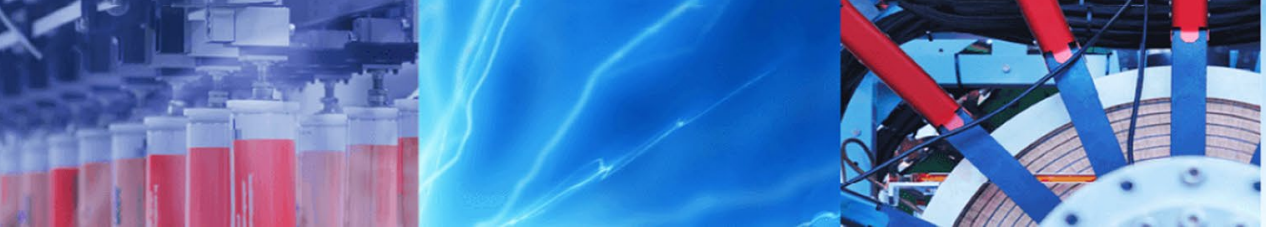

Research Article

\title{
Bio-inspired weighed quantum particle swarm optimization and smooth support vector machine ensembles for identification of abnormalities in medical data
}

\author{
T. P. Latchoumi ${ }^{1} \cdot$ T. P. Ezhilarasi ${ }^{2} \cdot$ K. Balamurugan ${ }^{3}$ (D)
}

C Springer Nature Switzerland AG 2019

\begin{abstract}
Knowledge mining is an emerging field where various patterns, rules, etc. can be generated which helps us in the analysis of the result. Medical information systems in modern hospitals and medical institutions become larger and larger, it causes great difficulties in extracting useful information for decision support. Especially, when traditional manual data analysis has become inefficient and methods for efficient computer-based analysis are indispensable. Decision support system is a suitable tool for medical application which helps in the extraction of useful data. A new hybrid model, weighted-quantum particle swarm optimization (WQPSO) for data clustering in sequence with smooth support vector machine (SSVM) is proposed for classification. The parameters considered to evaluate the clustering methods are intercluster distance, intra-cluster distance, and validation index. Experiments are performed in MATLAB and the performance analysis, comparisons are made with real-world datasets that are retrieved from UC Irvine Machine Learning Repository. The proposed approach overcomes the drawbacks of existing algorithms in terms of accuracy, performance and complexity. To validate the proposed algorithm, experiments are conducted on two data sets, i.e., liver disorders dataset and Wisconsin Breast Cancer Diagnosis (WBCD) dataset. The accuracy of proposed WQPSO-SSVM classification methodology is $83.76 \%$ for liver disorder, $98.42 \%$ for WBCD, $95.21 \%$ of mammography mass data. Among the considered algorithms such as WPSO-SVM, fuzzy, K-means, and fuzzy C-means methods, WQPSO-SSVM is found to yield a better convergence and provide an improved optimal solution.
\end{abstract}

Keywords Clustering classification · Knowledge data discovery $\cdot$ Quantum particle swarm optimization $\cdot$ Smooth support vector machine

\section{Introduction}

Biomedical datasets are complex datasets for clinical test results in microarray gene expression profiles and handling of this data is a challenging task. The improved methods are required to process such types of databases that are complex in nature. The data items within the cluster share some common properties, and data items in different clusters have their own distinct dissimilarity.
Improved particle swarm optimization (IPSO) with optimized employed parameters of support vector regression (SVR) has produced better recognition accuracy and generalization capacity than any other conventional model [1]. Enhanced particle swarm optimization algorithm is combined with SVM parameters to improve the capacity generalization and to guarantee its search in the global optimal solution instead of local optima [2]. Integration of the classification technique with enhanced cat swarm optimization (ECSO) and fruit fly optimization algorithm

K. Balamurugan, kbalan2000@gmail.com | 'Department of Computer Science and Engineering, VFSTR (Deemed to be University), Guntur, AP 522213, India. ²Department of Computer Science and Engineering, RAAK College of Engineering and Technology, Puducherry, India. ${ }^{3}$ Department of Mechanical Engineering, VFSTR (Deemed to be University), Guntur, AP 522213, India.

SN Applied Sciences (2019) 1:1137| https://doi.org/10.1007/s42452-019-1179-8

Received: 6 June 2019 / Accepted: 28 August 2019 / Published online: 3 September 2019 
(FFOA) was found to have predicted high accuracy and recognition in machine learning $[3,4]$.

The objective of data clustering is to analyze the distribution of data and to infer the relationship. Clustering technique is also referred to as unsupervised classification of data as it does not require prior knowledge of attributes and has no supervisor to do classification. It is generally categorized into hierarchical, partitioned, density-based and grid-based clustering and has been applied in several areas such as image segmentation, pattern recognition, marketing research, weather forecasting, etc. Classification organizes the unknown data into a pre-determined group consisting of pre-classified data objects. Clustering and classification made key contributions in identifying the patterns of interest in the medical discipline. K-means and FCM methods are used to divide the data elements with high convergence speed and $\mathrm{k}$ values. Various classification techniques like the nearest neighbor, Decision tree induction, rule-based, Bayesian and neural networkbased classifier are applied in diverse applications such as investment risk prediction [5], medical data classification [6], handwriting recognition, etc.

The medical dataset is specifically for alcoholic liver disorder and contains 345 data and 6 features of male individuals. The medical dataset is analyzed for inter-cluster, intracluster and validity index with three clustering techniques FCM, K-means, and PSO clustering. Knowledge Data Discovery (KDD) refers to the process of extracting useful knowledge from the database. Clustering and Classification are the data mining methods of KDD to discover and classify the patterns from input space which could help to derive knowledge over data. It involves an understanding of the application domain, creation of the target data set, data pre-processing, data reduction and projection, selection of data mining algorithms, searching for patterns, interpretation of patterns and finally consolidation of knowledge from the outcome of the above processes.

\section{Background}

Science discovers a huge number of critical diseases and causes. Computerized technology is becoming a great interest among the researchers to provide a better environment for the doctors and patients which helps in knowing the level of disease, treatments, etc. The available information should be readily manageable by the doctors so that, the patient can easily persuade about the disease level and its growth [7]. A Decision Support System (DSS) is a suitable tool for medical application which support both organizational and business activities with complete and reliable information [8]. Multiple heterogeneous biological data resources are readily available as a Data warehouse in the medical field. This biomedical dataset is widely used by the researchers to predict the abnormalities using weighed particle swarm optimization (WPSO), support vector machine (SVM), ECSO, IPSO, FFOA, etc. It is identified that DSS contains three types of databases along with responsibilities: Local database is responsible for gathering and storing the relational database of the knowledge acquired; model database assists with generating alternatives and storing, decision-making model and knowledge database model stores information and incorporates mathematical solvers [9]. Earlier studies reveal that the extracted data from the $\mathrm{UCl}$ repository is used by the PSO algorithm to optimize in terms of accuracy and helps in decision making by conducting the series of experiments [10].

Multivariate clustering data which is also called as model-based clustering deals with the dimensionality of data and helps in the selection of variables [11]. K-means is a clustering technique that uses the minimum number of iterations from the medical datasets and it is preferred as a suitable technique. To reduce the mathematical complexity of clustering of data, flocking based approach is used to classify the data from the datasets [12]. Data clustering is one of the prevalent and widely castoff data mining methods that gather alike data into groups based on the features of the data elements [13]. Data clustering is carried out in a supervised or unsupervised way by means of two main clustering methods: Partitional clustering and Hierarchical clustering. The number of clusters need not be quantified as a priority in hierarchical clustering, whereas cluster can be quantified in partitional approaches. Due to this reason, hierarchical methods cannot constantly set apart overlapping the clusters [14]. An improved PSO algorithm, namely rough particle swarm optimization algorithm (RPSO) was proposed to define the rough set of values based on the rough pattern's notions with upper and lower bounds [15]. This approach is efficiently used in data mining applications, especially in complex problems like association rule mining. There are numerous future research areas that deserve attention from the researchers working in the domain of multi-objective evolutionary algorithms (MOEAs)-based data mining algorithms [16]. adaptive network-based fuzzy inference system (ANFIS) is developed to forecast the market price based on the wavelet of time series by using its membership functions to tune the quantum particle swarm optimization (QPSO) [17]. The novel time warping (DTW)-wavelet transform (WT) method is used to extract the patterns automatically [18]. PSO provides better results in handling the arbitrary cost of nonlinear functions than the genetic algorithm (GA) [19]. PSO with fuzzy model improves the effectiveness of synthetic testing, resolution, and convergence for quality fitting and to solve computational time problems [20]. 
Multinomial Naïve Bayes model is proposed along with SVM and neural network (MLP) classifiers for designating the most popular varieties in biological applications [21].

An SVM model is developed and compared with the experimental observations on the compressive strength of brick-mortar masonry building at Kharagpur, India is found to be considered consistent [22]. PSO-SVM algorithm is used for group cell identification technology for biomedical application by providing improved clustering and corrected to $99.79 \%$ with good convergence compared to cross-validation algorithm [23]. WPSO-SSVM hybrid model was developed from PSO is useful in grouping the data of medical applications and used in the prediction on the level of severity of the diseases [24]. WPSOSSVM provide high accuracy and clear results to WBCD and Liver Disorder were the data sets are taken that helps to diagnosis the medical practitioner [25]. Developed fuzzy logic-PSO model convert unstructured data into a structured data format with a high level of accuracy, but it is a time-consuming method [26]. Similarly, Spatial Data Mining algorithm developed with PSO is used to predict disaster events in geographic application [27]. Kullback-Leibler divergence analytical method is used to measure the similarity between random variables in uncertain objects in discrete and continuous domains [28].

Multi-Elitist PSO (MEPSO) takes advantage of measuring the similarity of kernel-induced rather than measuring the sum-of-squares distance where most of the algorithms make [29]. PSO with velocity clamping and inertia weights (OvcPSO) is proposed to optimize the higher accuracy by controlling the direction, speed, and position of the particles [30]. Using PSO a set of machining condition are classified into some classes and the severity of the independent machining parameter for all the classes are easily accessed and measured in Abrasive Water Jet machine [31].

\section{Proposed methodology}

Generally, different objective functions are used to measure some intrinsic property of partitioning based on different views like the compactness of the clusters, a measure of cluster symmetry and density. But, considering a single objective function to measure the fitness of the cluster centroid may not be reflected in the results of partitioning. Multi-objective optimization (MOO) technique is an alternative solution to reflect effectiveness in clustering solutions. Data mining provides a promising solution in exploring knowledge concealed with the database. Data mining has been exploited in private and public sectors such as market segmentation, fraud detection, direct marketing, interactive marketing, market basket analysis, trend analysis, etc. The proposed approach is shown in Fig. 1. The features extracted from an image are being pre-processed to find the missing values. Preprocessed values are used to define the objective function that is clustered using WQPSO. It finds the fitness of each particle and global best positions of the particles. Set the global best and update position. SSVM ensembles for classification. The algorithms that have been proposed are verified by an extensive experimental study followed by strong theoretical analysis. The experimental results are found to have a correlation with the proposed model.

In QPSO, particles are moving in quantum space with particle state represented by wave function $\Psi(x, t)$ with probability distribution function (PDF) $\left|\Psi_{(\mathrm{x}, \mathrm{t})}\right|^{2}$. Being PDF, the particle appearing position and the changes in particle positions are represented as Eq. (1).

$\mathrm{x}_{\mathrm{i}}(\mathrm{t}+1)=\mathrm{P}_{\mathrm{i}}+\beta \mid$ Mbest $_{\mathrm{i}}-\mathrm{x}_{\mathrm{i}}(\mathrm{t}) \mid * \ln (1 / \mathrm{u})$,

$x_{i}(t+1)=P_{i}-\beta \mid$ Mbest $_{i}-x_{i}(t) \mid * \ln (1 / u)$,

$\beta$ denotes the contraction-expansion coefficient with $M$ representing the size of the population; Mbest (Mean best position) - mean value of the best position of all particles; $\mathrm{x}$-position of the particles; $\mathrm{t}$ - time convergence; $\mathrm{P}_{\mathrm{i}}$ - best position $P_{i}$ of the particle $i ; P_{i}$ and Mbest are found with Eqs. (2) and (3), respectively

$\mathrm{P}_{\mathrm{i}}=\varphi *$ pBest $_{\mathrm{i}}+(1-\varphi) *$ gBest $_{\mathrm{i}}$

Mbest $=\left(1 / \mathrm{M} \sum_{\mathrm{i}=1}^{\mathrm{M}} \mathrm{Pi} 1,1 / \mathrm{M} \sum_{\mathrm{i}=1}^{\mathrm{M}} \mathrm{Pi} 2, \ldots \ldots, 1 / \mathrm{M} \sum_{\mathrm{i}=1}^{\mathrm{M}} \mathrm{PiD}\right)$

$\mathrm{u}$ and $\varphi$ have random values distributed uniformly between [0, 1]; pBest—Local best particle; gBest—Global best particle; The steps for QPSO are 
Step 1: Initialize $\mathrm{M}$ as population size, $\mathrm{X}$ as particles position and $\mathrm{D}$ representing dimensions.

Step 2: Find each particles fitness

Step 3: Find pBest $\left(\mathrm{P}_{\mathrm{id}}\right)$ particle positions and then the gBest $\left(\mathrm{P}_{\mathrm{gd}}\right)$ amid particles.

While(condition)

Find Mbest positions using (3)

$$
\begin{aligned}
& \text { for } \mathrm{i} \text { ranging from } 1 \text { to } \mathrm{M} \\
& \text { for } \mathrm{j} \text { ranging from } 1 \text { to } \mathrm{D}
\end{aligned}
$$

update position of particles using (1)

end for

end while

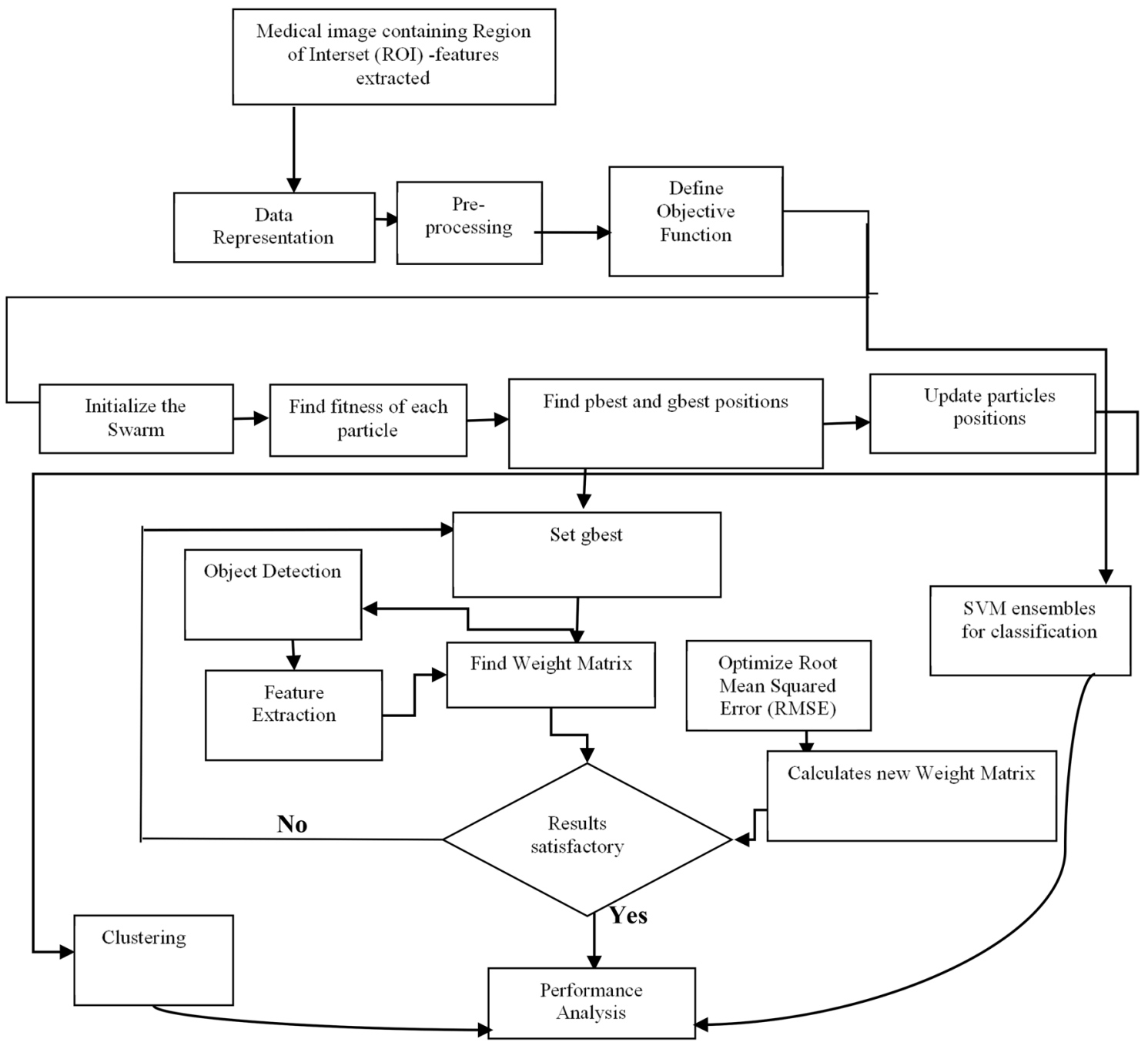

Fig. 1 Flow diagram of the proposed work 
Fig. 2 WPSO clustering

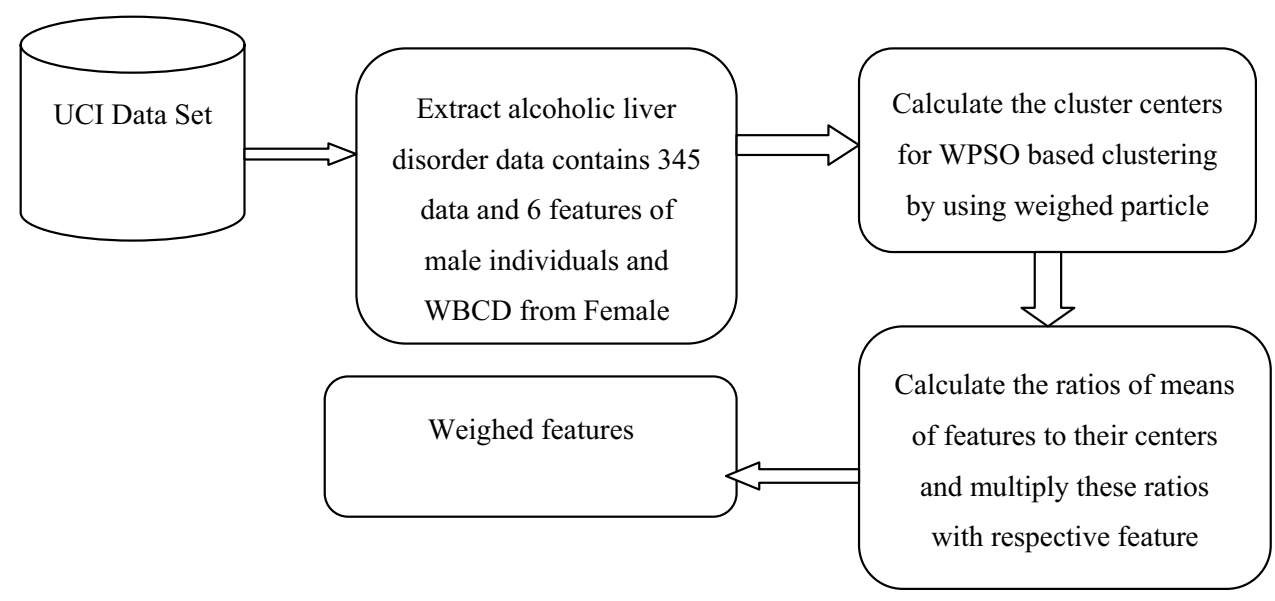

In QPSO, all particles are given equal importance but in WQPSO, fitness value is used for ranking of the particles. The particle that reaches its destination is considered to have high fitness. For helping the particles to escape from local minima prioritizing is done using weights.

The necessary steps for WQPSO are as follows

Step 1: Initialize $M$ as population size, $X[i]$ as particles position and $\mathrm{D}$ as dimensions and $\mathrm{P}[\mathrm{i}]$ equal $\mathrm{X}[\mathrm{i}]$;

Step 2: For the given population, update pBest positions.

Step 3: Set $P_{g}=\operatorname{Max}(p B e s t)$ and update the position of particles.

Step 4: Find $m$ best by Eq. (4).

mbest $=1 / M \sum_{i=1}^{M}$ ai1 Pi1, $1 / M \sum_{i=1}^{M}$ ai2Pi2, $\ldots \ldots, 1 / M \sum_{i=1}^{M}$ aiDPiD

$M$ is swarmed size, $a_{D}$ is the weight coefficient and $\mathrm{Pi}_{D}$ the particles best positions

Step 5: Update particles position X[i] to get global best positions

Step 6: Repeat steps 2-5 until the number of iterations.

\subsection{Feature weight assignment}

First assume a Medical case Library (ML) equal to $M L=(e 1$, e2, ..., eN). Each case in the library can be identified by an index of corresponding features. WQPSO clustering is shown in Fig. 2. In addition, each Medical case has an associated action that must be made for its current performance. The action can either be positive or negative which is to be decided based on the patient's symptoms. To make it more accurate collection of features i.e. $\mathrm{Fj}(j=1$ ... $\mathrm{n}$ ) is used to represent the cases and a variable $\mathrm{V}$ is used to denote the actions. The ith case ej in the library can be represented as $n+1$-dimensionalvector, i.e. ei $=\left(x_{i 1}, x_{i 2}\right.$, $\left.\ldots \ldots, x_{i n}, y_{i}\right)$. Where $x_{i j}$ corresponds to the value of feature $\mathrm{Fj}(\mathrm{j}=1 \ldots \mathrm{n})$ and $\mathrm{y}_{\mathrm{i}}$ corresponds to the action $(\mathrm{i}=1 \ldots \mathrm{n})$ to be taken.

Suppose that for each $\mathrm{j}(1 \leq \mathrm{j} \leq \mathrm{n})$ a weight $\mathrm{w}_{\mathrm{j}}\left(\mathrm{w}_{\mathrm{j}} \in[0,1]\right)$ has been assigned to the jth feature to indicate the importance of the feature, then for any pair of cases ep and eq in the library, a weighted distance metric is defined.

\subsection{Feature weight assignment algorithm}

The overall methodology is as follows:

Medical data set such as liver disorder or breast cancer is selected, and each case is indexed with a collection of features.

Each case is represented in the form of vector. Any two cases ep, eq, and weighted distance metric $\mathrm{d}_{\mathrm{pq}}^{(\mathrm{w})}$ using the Eq. (5).

$\mathbf{d}_{\mathbf{p q}}^{(\mathbf{w})}=\mathbf{d}^{(\mathbf{w})}\left(\mathbf{e}_{\mathbf{p}}, \mathbf{e}_{\mathbf{q}}\right)=\sum_{\mathbf{j}=1}^{\mathbf{n}} \mathbf{w}_{\mathbf{j}}^{2}\left(\mathbf{x}_{\mathbf{p j}}-\mathbf{x}_{\mathbf{q j}}\right)^{2^{1 / 2}}$

where xpj is the pth case with $j$ th feature and xqj is the qth case with the jth feature.

Using the above-weighted distance a similarity measure SM pq (w) is calculated using the Eq. (6)

$\mathbf{S M}_{\mathbf{p q}}^{(\mathbf{w})}=\frac{1}{1+\boldsymbol{\alpha} \mathbf{d}_{\mathbf{p q}}^{(\mathbf{w})}}$

where $a$ is a positive parameter

The weighted feature assignment algorithm is described as follows

Step 1: The parameter $a$, the learning rate $\eta$ are selected empirically.

Step 2: Initialize wj with random values in $[0,1]$.

Step 3: Compute wj for each j using Eq. (7). 
Step 4: Calculate evaluation function $\mathrm{E}(\mathrm{w})$ using Eq. (8). Step 5: Update wj with wj $+\Delta$ wj for each $\mathbf{j}$.

Step 6: Repeat steps 3 and 4 until convergence until the value of $E$ becomes less than or equal to a given threshold or until the number of iterations exceeds a certain predefined number.

Step 7: Use the final wj as the weighted feature for PSOclustering.

$$
\Delta \mathbf{w}_{\mathbf{j}}=-\eta \frac{\partial \mathbf{E}}{\partial \mathbf{w}_{\mathbf{j}}}
$$

where $\eta$ is the learning rate

$\mathbf{E}(\mathbf{w})=2 \times\left[\frac{\sum_{\mathbf{p q}} \sum_{\mathbf{q}<\mathbf{p}} \mathbf{S M}_{\mathbf{p q}}^{(\mathbf{w})}\left(1-\mathbf{S M}_{\mathbf{p q}}^{(1)}\right)+\mathbf{S M}_{\mathbf{p q}}^{(1)}\left(1-\mathbf{S M}_{\mathbf{p q}}^{(\mathbf{w})}\right)}{\mathbf{N}(\mathbf{N}-1)}\right]$

where SMpq is the similarity measure; $\mathrm{N}$ is the number of data in the dataset.

To calculate $\frac{\partial \mathbf{E}}{\partial \mathbf{w}_{\mathbf{j}}}$ the following Eqs. (9), (10) and (11) are used:

\section{Table 1 Parameters value}

\begin{tabular}{lll}
\hline Parameter setting & Liver disorders & $\begin{array}{l}\text { Wisconsin } \\
\text { breast } \\
\text { cancer }\end{array}$ \\
\hline a & 0.6 & 0.6 \\
Learning rate $\eta$ & 0.05 & 0.05 \\
\hline
\end{tabular}

$\frac{\partial \mathbf{E}(\mathbf{w})}{\partial \mathbf{w}_{\mathbf{j}}}=\frac{2 *\left[\sum_{\mathbf{p q}} \sum_{\mathbf{q}<\boldsymbol{p}}\left(1-2 \mathbf{S M}_{\mathbf{p q}}^{(1)} \cdot \frac{\partial \mathbf{S M}_{\mathbf{p q}}^{(\mathbf{w})}}{\partial \mathbf{d}_{\mathbf{p q}}^{(\mathbf{w})}} \cdot \frac{\partial \mathbf{d}_{\mathbf{p q}}^{(\mathbf{w})}}{\partial \mathbf{w}_{\mathbf{j}}}\right)\right]}{\mathbf{N} *(\mathbf{N}-1)}$

$\frac{\partial \mathbf{S} \mathbf{M}_{\mathbf{p q}}^{(\mathbf{w})}}{\partial \mathbf{d}_{\mathbf{p q}}^{(\mathbf{w})}}=\frac{-\boldsymbol{\alpha}}{\left(1+\boldsymbol{\alpha} \cdot \mathbf{d}_{\mathbf{p q}}^{(\mathbf{w})}\right)^{2}}$

$\frac{\partial \mathbf{d}_{\mathbf{p q}}^{(\mathbf{w})}}{\partial \mathbf{w}_{\mathbf{j}}}=\frac{\mathbf{w}_{\mathbf{j}} \mathbf{X}_{\mathbf{j}}^{2}}{\left(\sum_{\mathbf{j}=1}^{\mathbf{n}} \mathbf{w}_{\mathbf{j}}^{2} \mathbf{X}_{\mathbf{j}}^{2}\right)^{\left(\frac{1}{2}\right)}}$

The parameter $a$ and $\eta$ which are used for assigning a weight for each feature, selected on an experimental basis are shown in Table 1

In QPSO, all particles are given equal importance but in WQPSO, fitness value is used for ranking of the particles. The particle that reaches its destination is considered to

Table 2 Comparison of inter, intra and validity index with FCM, K-means, and WQPSO for WBCD

\begin{tabular}{llrr}
\hline Measures & FCM & K-means & WQPSO \\
\hline Inter cluster & 708.561 & 713.944 & 941.771 \\
Intra cluster & NA & 11.457 & 0.292 \\
Validity & NA & 0.016 & 0.003 \\
\hline
\end{tabular}

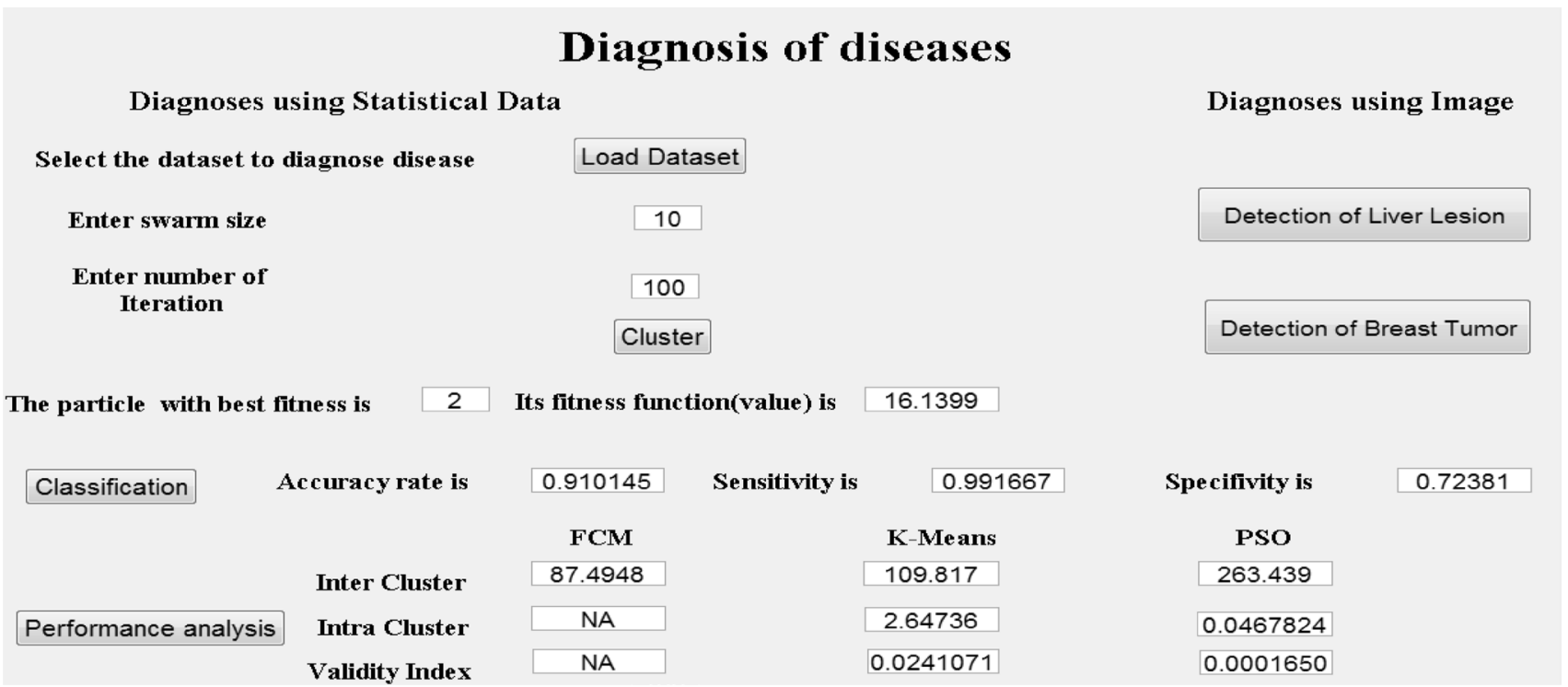

Fig. 3 Performance analysis of liver tumor chart obtained from MATLAB clustering tool box 


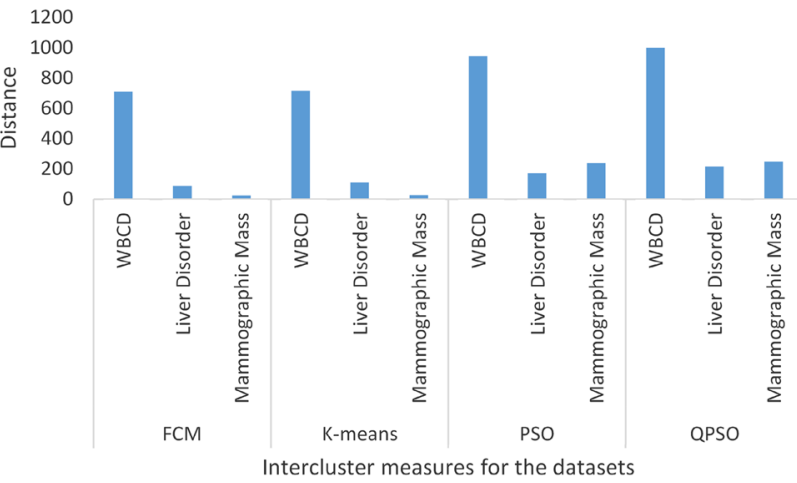

Fig. 4 Comparison of inter-cluster distance with FCM, K-means, PSO, and QPSO for breast cancer (WBCD), liver disorder and mammographic mass dataset

have high fitness. For helping the particles to escape from local minima prioritizing is done using its weights.

\subsection{Weighed quantum particle swarm optimization (WQPSO)}

The necessary steps for WQPSO are as follows
Table 4 Confusion matrix

\begin{tabular}{lll}
\hline & $\mathrm{p}^{\prime}$ (predicted) & $\mathrm{n}^{\prime}$ (predicted) \\
\hline $\mathrm{p}$ (actual) & True positive & False negative \\
$\mathrm{n}$ (actual) & False positive & True negative \\
\hline
\end{tabular}

Step 1: Initialize $M$ as population size, $X[i]$ as particles position and $\mathrm{D}$ as dimensions and $\mathrm{P}[\mathrm{i}]$ equal $\mathrm{X}[\mathrm{i}]$; Step 2: For the given population, update pbest positions.

Step 3: Set $\mathrm{Pg}=\operatorname{Max}($ pbest) and update the position of particles.

Step 4: Find $m$ best from Eq. (4).

mbest $=1 / M \sum_{i=1}^{M}$ ai1Pi1, $1 / M \sum_{i=1}^{M}$ ai2Pi2, .... 1/M $\sum_{i=1}^{M}$ aiDPiD

Step 5: Update particles position X[i] to get global best positions

Step 6: Repeat steps $2-5$ until the number of iterations.

Table 3 Comparison of inter, intra and validity index with FCM, K-means PSO and WQPSO for breast cancer (WBCD), Liver disorder and mammographic mass dataset

\begin{tabular}{|c|c|c|c|c|c|c|c|c|c|}
\hline \multirow[t]{2}{*}{ Measures } & \multicolumn{3}{|l|}{ K-means } & \multicolumn{3}{|l|}{ PSO } & \multicolumn{3}{|l|}{ WQPSO } \\
\hline & WBCD & Liver Disorder & $\begin{array}{l}\text { Mammo } \\
\text { graphic } \\
\text { Mass }\end{array}$ & WBCD & Liver Disorder & $\begin{array}{l}\text { Mammo } \\
\text { graphic } \\
\text { Mass }\end{array}$ & WBCD & Liver Disorder & $\begin{array}{l}\text { Mammo } \\
\text { graphic } \\
\text { mass }\end{array}$ \\
\hline Inter Cluster & 713.941 & 109.822 & 26.912 & 941.771 & 172.302 & 237.602 & 998.422 & 215.471 & 248.442 \\
\hline Intra Cluster & 11.457 & 2.647 & 0.394 & 0.292 & 0.085 & 0.004 & 0.188 & 0.006 & 0.004 \\
\hline Validity & 0.016 & 0.024 & 0.016 & 0.004 & 0.005 & $1 \mathrm{E}-04$ & 0.002 & 0.005 & 0 \\
\hline
\end{tabular}

Fig. 5 Fitness value of QPSO chart obtained from MATLAB clustering tool box

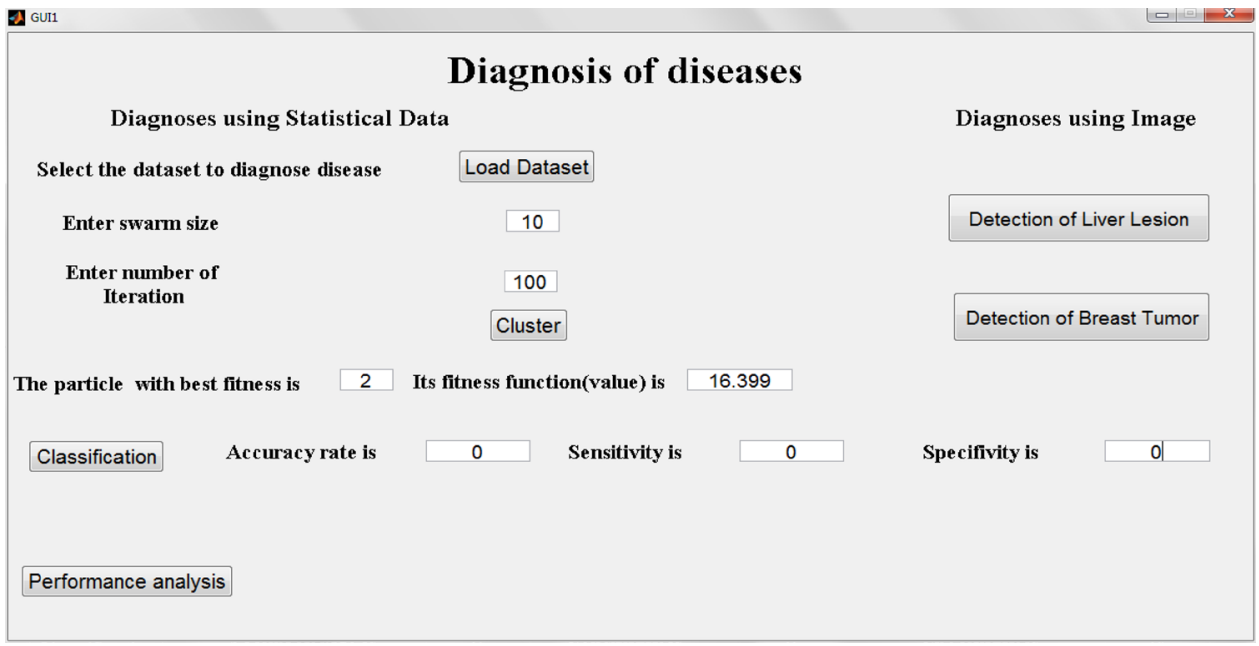

SN Applied Sciences A SPRINGER NATURE journa 


\section{Experimental results}

The medical dataset of liver disorder for the present work is obtained from the database repository. This dataset is specifically for alcoholic liver disorder and contains 345 data and 6 features of male individuals. The medical data set is analyzed for inter-cluster, intra-cluster and validity index with three clustering techniques FCM, K-means, and WQPSO clustering. The inter-cluster distance of any two clusters should be high.

Figure 3 shows the Performance analysis of liver tumor developed. The inter-cluster distance of any two clusters should be high. As seen in Table 2, the distance between two clusters for FCM is 708.561, for K-means is 713.944 and for WQPSO is 941.771 . Intra-cluster means the compactness of a cluster and its value should be as low as possible. As seen in Table 2 for WQPSO is 0.292 and for K-means is 11.457. Validity should be minimized for a better cluster and it could be best for PSO with 0.00031 .

The WBCD, mammographic mass and liver disorder datasets are obtained from database. WQPSO clustering is applied to the datasets and compared with K-means, PSO, and QPSO in terms of inter-cluster distance and it is shown in Fig. 4.

The inter-cluster distance of any two clusters should be high which is best for QPSO as seen in Table 3. Intra-cluster means the compactness of a cluster and its value should be least as possible and is again found to be best for QPSO.

Figure 5 shows the fitness value for QPSO is performed through a MATLAB tool. This shows the fitness value of

Table 5 Calculation formula for sensitivity, specificity, accuracy, and precision

Table 6 SSVM ensembles with WQPSO and obtained results

\begin{tabular}{ll}
\hline Sensitivity & $\frac{T P}{T P+F N}$ \\
Specificity & $\frac{T N}{T N+F P}$ \\
Accuracy & $\frac{T P+T N}{T P+T N+F P+F N}$ \\
Precision & $\frac{T P}{F P+T P}$ \\
\hline
\end{tabular}

WBCD, Liver Disorder and Mammographic mass diseases using QPSO.

The clustered groups are classified with SVM ensembles and the obtained results are shown in Table 4. The accuracy of SVM classifier is calculated by using the confusion matrix.

In the Confusion Matrix, for each cell in the matrix, we have fields as True Positives, False Positives, False Negatives, and True Negatives.

- True Positive (TP)—Abnormal people correctly identified as Abnormal

- True Negative (TN)_Normal people correctly identified as Normal

- False Positive (FP)—Normal people incorrectly identified as Abnormal

- False Negative (FN)-Abnormal people incorrectly identified as Normal

The metrics used in classification are calculated is shown in Table 5.

Sensitivity The sensitivity of the feature extraction and the feature classification is dictated by taking the proportion of several true positives to the whole of a true positive and false negative.

Specificity The specificity of the feature extraction and the feature classification can be assessed by taking the connection of several true negatives to the consolidated true negative and the false positive.

Accuracy The accuracy of feature extraction and the feature classification can be figured condition (7) by taking the proportion of true esteems show in the populace. Precision Identifies the positive predictive value.

The clustered groups are classified with SVM ensembles and obtained results are presented in Table 6 .

\begin{tabular}{|c|c|c|c|c|c|c|c|c|c|}
\hline \multirow[t]{2}{*}{ Methods } & \multicolumn{3}{|c|}{ WBCD in \% } & \multicolumn{3}{|c|}{ Liver disorder in \% } & \multicolumn{3}{|c|}{$\begin{array}{l}\text { Mammographic Mass } \\
\text { in } \%\end{array}$} \\
\hline & $A$ & $\mathrm{Se}$ & $S p$ & $A$ & $\mathrm{Se}$ & $S p$ & $A$ & $\mathrm{Se}$ & $S p$ \\
\hline RULES-4 & 94.8 & 96.4 & 92.5 & 55.9 & 56.7 & 54.5 & 78.1 & 79.5 & 75.6 \\
\hline C4.5 & 96.8 & 97.1 & 94.5 & 65.6 & 66.8 & 64.9 & 81.1 & 84.5 & 79.6 \\
\hline Naive Bayes & 97.3 & 98.5 & 96.2 & 63.4 & 66.5 & 61.2 & 83.4 & 86.6 & 82.3 \\
\hline SVMwithGP & 96.7 & 98.4 & 94.9 & 69.7 & 71.7 & 65.7 & 83.7 & 85.5 & 81.1 \\
\hline MLP & 97.2 & 98.5 & 96.2 & 73.1 & 74.6 & 72.5 & 84.8 & 87.6 & 82.5 \\
\hline $\mathrm{CBR}+\mathrm{PSO}$ & 97.4 & 98.5 & 96.4 & 76.8 & 77.7 & 73.7 & 85.3 & 87.6 & 83.4 \\
\hline PSO + SVM & 98.4 & 99.3 & 97.3 & 83.2 & 86.2 & 77.2 & 95.2 & 97.6 & 93.5 \\
\hline $\begin{array}{l}\text { Proposed method } \\
\text { (WQPSO+SSVM) }\end{array}$ & 98.7 & 99.4 & 97.8 & 84.7 & 87.9 & 79.5 & 96.7 & 98.1 & 94.6 \\
\hline
\end{tabular}

A accuracy, Se sensitivity, Sp specificity 


\section{Conclusion}

Standard PSO has a minimum number of control parameters and it can easily confine into the local optima. The proposed WQPSO-SSVM algorithm is used to improve the classification accuracy of medical datasets and the results are found to outperform all the present state of-art classifiers existing in the literature. To eradicate the issue in the existing system, the proposed WQPSO-SSVM algorithm has improvement than the existing PSO algorithm in terms of quantum theory and weighted mean best position calculations. WQPSO-SSVM algorithm will balance the global and local search in terms of utilizing global search space and avoids local optima using high similar intra-class clusters. The accuracy of proposed WQPSO-SSVM classification methodology is $84.7 \%$ for a liver disorder, $98.7 \%$ for WBCD, $96.7 \%$ of mammography mass data.

A hybridized WQPSO-SSVM ensembles technique is proposed to increase the accuracy of classification of biomedical datasets. PSO converges at the local optimum point in many situations and it requires velocity and position vector to keep the record of particles velocity and positions. But QPSO and WQPSO do not require velocity vector, where particles have quantum behavior and ensure global convergence. WQPSO maintains particles rank according to their fitness and this unique nature helps to reach the goal faster and assure better convergence.

Other notable advantages of the proposed approach are:

- Reduction in the complexity of the data set.

- Speed up the clustering computation time

- Increased accuracy and reduction in total run time.

The limitation of the proposed work is an increase of computational complexity due to the usage of weighing optimization strategy. Some studies yet to be conducted to reduce the computational complexity.

\section{Compliance with ethical standards}

Conflict of interest The authors declare that they have no conflict of interest.

\section{References}

1. Yu Y, Li Y, Li J (2015) Forecasting hysteresis behaviors of magnetorheological elastomer base isolator utilizing a hybrid model based on support vector regression and improved particle swarm optimization. Smart Mater Struct. https://doi. org/10.1088/0964-1726/24/3/035025
2. Yu Y, Zhang C, Gu X, Cui Y (2018) Expansion prediction of alkaliaggregate reactivity-affected concrete structures using a hybrid soft computing method. Neural Comput Appl. https://doi. org/10.1007/s00521-018-3679-7

3. Yu Y, Li W, Li J, Nguyen TN (2018) A novel optimized self-learning method for compressive strength prediction of high-performance concrete. Constr Build Mater 184:229-247

4. Yu Y, Li Y, Li J, Gu X (2016) Self-adaptive step fruit fly algorithm optimized support vector regression model for dynamic response prediction of magnetorheological elastomer base isolator. Neurocomputing 211:41-52

5. Bodaghi A, Teimourpour B (2018) The detection of professional fraud in automobile insurance using social network analysis. Social and information networks. Dissertation, Tarbiat Modares University, Iran

6. PaulAK ShillPC, Rabin MRI, Murase K (2018) An adaptive weighted fuzzy rule-based system for the risk level assessment of heart disease. Appl Intell 48:1739-1756

7. Mohammed MA, Ghani MKA, Arunkumar N, Hamed Rl, Mostafa SA, Abdullah MK, Burhanuddin MA (2018) Decision support system for nasopharyngeal carcinoma discrimination from endoscopic images using an artificial neural network. J Supercomput. https://doi.org/10.1007/s11227-018-2587-z1-19

8. Sharma P, Itu LM, Flohr T, Comaniciu D, (2019) U.S. Patent Application No. 10/176,896

9. Zhao L, Long Y (2019) An improved PSO algorithm for the classification of multiple power quality disturbances. J Inf Process Syst 1:116-126

10. Alswaitti M, Albughdadi M, Isa NAM (2018) Density-based particle swarm optimization algorithm for data clustering. Expert Syst Appl 91:170-186

11. Fop M, Murphy TB (2018) Variable selection methods for modelbased clustering. Stat Surv 12:18-65

12. PambudiEA AndonoPN, Pramunendar RA (2018) Image segmentation analysis based on K-means PSO by using three distance measures. ICTACT J Image Video Process 9:1821-1826

13. TarkhanehO IsazadehA, Khamnei HJ (2018) A new hybrid strategy for data clustering using cuckoo search based on Mantegna levy distribution, PSO and k-means. Int J Comput Appl Technol 2:137-149

14. Alatas B, Akin E (2008) Rough particle swarm optimization and its applications in data mining. Soft Comput 12:1205-1208

15. Von L, Anatole $O$ (2018) Quantum machine learning in chemical compound space. Angewandte Chemie Int Ed 16:4164-4169

16. Anirban M, Ujjwal M, Sanghamitra B, Carlos C (2014) Survey of multi-objective evolutionary algorithms for data mining: part II. J IEEE Trans Evol Comput 18:20-35

17. Bagheri A, Mohammadi PH, Akbari M (2014) Financial forecasting using ANFIS networks with quantum-behaved particle swarm optimization. Expert Syst Appl 41:6235-6250

18. Jia PF, Tian FC, Fan S, He QH, Feng JW, Yang SX (2014) A novel sensor array and classifier optimization method of the electronic nose based on enhanced quantum-behaved particle swarm optimization. Sens Rev 34:304-311

19. Boeringer DW, Werner DH (2004) Particle swarm optimization versus genetic algorithm for phased array synthesis. IEEE Trans Antennas Propag 3:771-779

20. Ouali MA, Ghanai M, Chafaa K (2018) A new type-2 fuzzy modeling and identification for electrophysiological signals: a comparison between PSO, BBO, FA, and GA approaches. Int J Model Ident Control 2:163-184

21. Sharma AK, Sudesh KP, Mohammed A (2014) A comparative study between naive Bayes and neural network (MLP) classifier for spam email detection. Int J Comput Appl 2:12-16 
22. Mishra M, Bhatia AS, Maity D (2019) Support vector machine for determining the compressive strength of brick-mortar masonry using NDT data fusion (case study: Kharagpur, India). SN Appl Sci 6:564. https://doi.org/10.1007/s42452-019-0590-5

23. Wang Y, Meng X, Zhu L (2018) Cell group recognition method based on adaptive mutation PSO-SVM. Cells 9:135

24. Chang PC, Lin JJ, Liu CH (2012) An attribute weight assignment and particle swarm optimization algorithm for medical database classifications. Comput Methods Programs Biomed 107:382-392

25. Latchoumi TP, Latha P (2017) Abnormality detection using weighed particle swarm optimization and smooth support vector machine. Biomed Res 28:4749-4751

26. Ravikumar K, Rajiv KA (2018) Spatial data mining for prediction of natural events and disaster management based on fuzzy logic using hybrid PSO. TAGA J 14:858-878

27. Baozhi Q, Xiaofeng C (2016) Clustering boundary detection for high dimensional space based on space inversion and Hopkins statistics. J Knowl Based Syst 98:216-225
28. Bin J, Jian P, Yufei T, Xuemin L (2013) Clustering uncertain data based on probability distribution similarity. J IEEE Trans Knowl Data Eng 25:751-763

29. Das S, Abraham A, Konar A (2008) Automatic kernel clustering with a multi-elitist particle swarm optimization algorithm. Pattern Recognit Lett 29:688-699

30. Shahzad F, Masood S, Khan NK (2014) Probabilistic oppositionbased particle swarm optimization with velocity clamping. Knowl Inf Syst 39:703-737

31. Latchoumi TP, Balamurugan K, Dinesh K, Ezhilarasi TP (2019) Particle swarm optimization approach for waterjet cavitation peening. Measurement 141:184-189

Publisher's Note Springer Nature remains neutral with regard to jurisdictional claims in published maps and institutional affiliations. 